\title{
CT artifacts after contrast media injection in chest imaging: evaluation of post-processing algorithms, virtual monoenergetic images and their combination for artifact reduction
}

\author{
Amit Gupta ${ }^{1,2}$, Verena Carola Obmann ${ }^{1,2,3}$, Michelle Jordan $^{1,2}$, Simon Lennartz ${ }^{4}$, \\ Markus Michael Obmann ${ }^{5}$, Nils Große Hokamp ${ }^{1,2,4}$, David Zopfs ${ }^{4}$, Lenhard Pennig ${ }^{4}$, Gina Fürtjes ${ }^{6}$, \\ Nikhil Ramaiya ${ }^{1,2}$, Robert Gilkeson ${ }^{1,2}$, Kai Roman Laukamp ${ }^{1,2,4}$ \\ ${ }^{1}$ Department of Radiology, University Hospitals Cleveland Medical Center, Cleveland, OH, USA; ${ }^{2}$ Department of Radiology, Case Western \\ Reserve University, Cleveland, OH, USA; ${ }^{3}$ Department of Diagnostic, Interventional and Pediatric Radiology, Inselspital, Bern University Hospital, \\ University of Bern, Switzerland; ${ }^{4}$ Institute for Diagnostic and Interventional Radiology, Faculty of Medicine and University Hospital Cologne, \\ University of Cologne, Cologne, Germany; ${ }^{5}$ Clinic of Radiology and Nuclear Medicine, University Hospital Basel, University of Basel, Basel, \\ Switzerland; ${ }^{6}$ Center for Neurosurgery, Faculty of Medicine and University Hospital Cologne, University of Cologne, Cologne, Germany
}

Correspondence to: Kai Roman Laukamp. Department of Radiology, University Hospitals Cleveland Medical Center, 11000 Euclid Ave, Cleveland, OH 44106, USA. Email: kai.laukamp@uk-koeln.de.

Background: After injection into a brachial vein, high contrast media concentration in axillary and subclavian veins can cause artifacts that impair diagnostic utility. This study assessed artifact reduction by artifact-reduction-algorithms (ARA) and virtual-monoenergetic-images (VMI), as well as their combination $\left(\mathrm{VMI}_{\mathrm{ARA}}\right)$ compared to conventional CT-images (CI).

Methods: Forty-six spectral-detector-CT (SDCT) examinations of patients that received ARAreconstructions due to perivenous-artifacts were included in this retrospective study. CI, ARA, VMI, and $\mathrm{VMI}_{\mathrm{ARA}}$ (range: 70-200 keV) were reconstructed. Objective analysis was performed with ROI-based assessment of mean and standard deviation of attenuation (HU) in hypo- and hyperdense artifacts and impaired muscle and arteries as well as artifact-free reference-tissue. Extent of artifact reduction, assessment of surrounding soft tissue and vessels, and appearance of new artifacts were rated visually by two radiologists. Results: Hypo- and hyperdense artifacts showed significant improvement as evidenced by decreasing attenuation differences between artifact impaired and artifact-free reference tissue in ARA, VMI $\geq 80 \mathrm{keV}$, and $\mathrm{VMI}_{\mathrm{ARA}}$ between 70-200 keV (e.g., CI/ARA/VMI ${ }_{100 \mathrm{keV}} / \mathrm{VMI}_{\mathrm{ARA} 100 \mathrm{keV}}$ : hypodense artifacts, (-)264.8 $\pm 150.9 /$ (-)87.1 $\pm 78.9 /(-) 48.6 \pm 64.6 / 9.9 \pm 63.9 \mathrm{HU} ; \mathrm{P}<0.001)$; hyperdense artifacts, $164.2 \pm 51.1 / 82.1 \pm 73.2 / 7.9 \pm 34.7 /$ $(-) 17.3 \pm 50.7 \mathrm{HU} ; \mathrm{P}<0.001)$. Artifacts impairing surrounding muscle and arteries were also reduced by all three approaches. In visual assessment, $\mathrm{ARA}, \mathrm{VMI} \geq 100 \mathrm{keV}$, and $\mathrm{VMI}_{\mathrm{ARA}}$ between $70-200 \mathrm{keV}$ also showed significant artifact reduction and improved assessment; however, for assessment of arteries improvement was not significant using ARA alone. New artifacts were reported, particularly at higher keV-values.

Conclusions: In presence of perivenous-artifacts, ARA, VMI and their combination allow for significant artifact reduction; however, their combination and VMI as a standalone approach yielded best results and should therefore be used, if available.

Keywords: X-ray computed tomography; artifacts; contrast media; algorithms; dual-energy CT; spectral-detector CT (SDCT)

Submitted Mar 13, 2020. Accepted for publication Aug 28, 2020.

doi: 10.21037/qims-20-435

View this article at: http://dx.doi.org/10.21037/qims-20-435 


\section{Introduction}

Multidetector CT acquisitions require specialized contrast media injection protocols. Contrast media is usually injected as concentrated boluses (1). As contrast media is strongly attenuating in CT-imaging, it can thereby like metal cause severe artifacts (2-7). These artifacts commonly appear as hypo- and hyperdense areas surrounding the highly attenuating material. Artifacts from highly attenuating material results from (I) beam-hardening [preferential absorption of low energetic photons $(8,9)]$, (II) photon starvation [complete absorption of all photons $(5,9)]$, and (III) scatter artifacts [resulting from greater attenuation differences, e.g., soft tissue and contrast media (6)]. Contrast media is mostly injected via an upper extremity venous access; therefore, these artifacts are most pronounced around axillary and subclavian veins. Here they may impair assessment of surrounding structures, including muscle, lymph nodes, fat, and adjacent arterial vessels (2-4,7). Lung and mediastinal structures are less commonly impaired by these kind of artifacts as contrast media becomes more diluted in larger vessels closer to the heart $(2,4,7,10)$.

Dual-layer spectral-detector CT (SDCT) separately detects low and high energy photons. This enables calculation of virtual monoenergetic images (VMI) that correspond to images that would result from true monoenergetic $\mathrm{x}$-ray acquisitions (11-13). High keV VMI reduce artifacts since high-keV values are less susceptible to beam hardening. This has been shown for metal materials (14-17) and recently also for artifacts from contrast media $(2,18)$. Dedicated artifact reduction algorithms (ARA) have been developed to reduce artifacts from highly attenuating material in conventional single-energy images $(16,19)$. They are primarily designed for larger metal implants, e.g., total hip replacements $(19,20)$. Still, in recent studies, ARA were also found to confer robust artifact reduction for smaller implants $(21,22)$. This might suggest that ARA could also be applicable for other highly attenuating material, i.e., contrast media (20). Further, recent studies showed that the combination of ARA and VMI is often the most effective approach for artifact reduction $(21,23)$. However, the effectiveness of this combined approach has not been investigated for reduction of artifacts resulting from contrast media injection.

The purpose of this study was to investigate the value of ARA in combination with VMI $\left(\mathrm{VMI}_{\mathrm{ARA}}\right)$ for reduction of artifacts from contrast media after upper extremity intravenous injection in chest imaging.

\section{Methods}

This retrospective study was approved by the local institutional review board and in accordance with the Health-Insurance-Portability and AccountabilityAct. Informed written consent was waived. Inclusion criteria were applied by a radiologist with 4 years of experience in chest imaging and as follows: (I) age $\geq 18$ years, (II) contrast enhanced thoracoabdominal staging and restaging examinations in portal-venous phase between June 2018 and March 2019, (III) presence of artifacts that impaired diagnostic assessment from high contrast media concentration in axillary and subclavian veins, and (IV) availability of ARA in addition to conventional CT images (CI) and VMI. Contrary to CI and VMI, ARA are not retrospectively available and needed to be additionally applied if perivenous artifacts compromised image assessment of circumjacent structures. Applying the abovementioned criteria, a total of 46 non-consecutive patients were eligible for study inclusion.

\section{Imaging protocol}

Examinations were conducted on a clinical SDCT (IQon, Philips Healthcare) with patient in a head-first supine position with raised arms. Iodinated contrast media (Optiray 350 , Guerbet) was injected through an antecubital vein. Amount of contrast was adapted to body weight $(1.5 \mathrm{~mL} / \mathrm{kg}$, maximum total amount applied per patient was set to $140 \mathrm{~mL}$ ). Flow rate was $2.0 \mathrm{~mL} / \mathrm{s}$. Acquisition began 70 seconds after contrast media injection started. Scan parameters were set as follows: collimation $64 \mathrm{~mm} \times$ $0.625 \mathrm{~mm}$, rotation-time 0.33 seconds, pitch 0.98 , matrix $512 \times 512$. Tube voltage was $120 \mathrm{kVp}$ and automatic tube current modulation (dose right, Philips Healthcare) was used with a dose right index of 15 , employing both $\mathrm{Z}$-modulation and 3D-dose modulation.

Conventional CT-images (iDose ${ }^{4}$, level 3; referred to as CI), ARA (O-MAR; Philips Healthcare), VMI (Spectral $\mathrm{B}$, level 3, range: $70-200 \mathrm{keV}$, increment of $10 \mathrm{keV}$ ), and the combination of ARA and VMI (Spectral B, level 3 and O-MAR, range: 70-200 keV, increment of $10 \mathrm{keV}$ ) were reconstructed. Slice thickness was set to $3 \mathrm{~mm}$.

\section{Objective analysis}

Image assessment was conducted by using regions of 
interest (ROI) with a consistent size of $100 \mathrm{~mm}^{2}$. ROI size could possibly be reduced to avoid unrepresentative tissue. First, ROI were placed on CI and then copied to ARA, VMI and $\mathrm{VMI}_{\mathrm{ARA}}$ using the vendor's proprietary image viewer (IntelliSpace Portal v9, Philips Healthcare). ROI were placed in the most pronounced hypo- and hyperdense artifacts as well as corresponding artifact-free reference tissue, e.g., when a hyperdense artifact obscured muscle, the corresponding reference tissue was contralateral artifactfree muscle; this procedure was applied individually for each artifact. ROI were also placed in surrounding artifact impaired muscle and arteries as well as corresponding artifact-free reference tissue. Mean and standard deviation of attenuation were recorded. Standard deviation in artifact impaired muscle and arteries were regarded representative for image noise and indicative of artifact burden (24).

As was done for previous studies $(21,25,26)$, corrected attenuation for hypo- and hyperdense artifacts as well as artifact impaired muscle and arteries was defined as the difference between attenuation in artifact impaired and nonaffected reference tissue. This method accounts for changes in attenuation at different $\mathrm{keV}$ values for VMI to minimize any bias and detect real artifact reduction, e.g., with higher $\mathrm{keV}$ values attenuation in VMI of muscle and artery decreases whereas attenuation of fat increases (11). For artifact impaired muscle and artery, we also calculated corrected image noise $(21,25,26)$, as the difference between image noise in artifact impaired and artifact-free reference tissue to correct for general lower image noise in high keV VMI (11).

\section{Visual analysis}

Two board-certified fellowship trained radiologists with eight and nine years of experience conducted the visual analysis. They evaluated extent of hypo- and hyperdense artifacts as well as diagnostic assessment using 5-point Likert-scales (Table 1). Also, introduction of overcorrection or new artifacts were rated on a tertiary scale (Table 1). Overcorrection was defined as artifacts that occurred in the same location as the original artifacts but that did not additionally impair diagnostic assessment compared to CI. New artifacts were defined as artifacts in the same or other locations as the original artifacts that further impaired diagnostic assessment compared to CI. Both readers reported the optimal $\mathrm{keV}$ value for overall diagnostic assessment considering extent of artifact, introduction of overcorrection and new artifacts as well as loss of soft tissue contrast, which is a known effect of high $\mathrm{keV}$ VMI $(11,21)$.
Readings were conducted on CI, ARA, VMI, and $\mathrm{VMI}_{\mathrm{ARA}}$. VMI and $\mathrm{VMI}_{\mathrm{ARA}} \mathrm{keV}$ values were 70, 100, 130,160 , and $200 \mathrm{keV}$. Larger increments compared to the objective assessment were used to allow detection of relevant changes in image assessment that might be overlooked by evaluating images with smaller increments that might appear too similar. Image parameters were as follows: slice thickness $3 \mathrm{~mm}$, axial plane, soft tissue window (window-level: 60, window-width: 350). Readers were allowed to adapt window settings.

The board-certified fellowship trained radiologists with nine years of experience also assessed if the ARA, VMI and $\mathrm{VMI}_{\mathrm{ARA}}$ could reveal any additional axillary or subclavian lymph nodes that were obscured by artifacts in CI.

\section{Statistical analysis}

Statistical analysis was conducted using JMP-Software (V14, SAS-Institute). Quantitative results are presented as mean \pm standard deviation and qualitative results are given by median and 10/90-percentile. Shapiro-Wilk test was used to test for normal distribution. Wilcoxon-test with Steel-adjustment for multiple comparisons and Wilcoxon signed-rank were used to test for any significant difference. Statistical significance was set to $\mathrm{P}<0.05$. Interreaderagreement was assessed using intraclass-correlationcoefficient (ICC) and interpreted as proposed earlier $(27,28)$; agreement being poor $<0.40$; fair $0.40-0.59$; good $0.60-0.75$; and excellent $0.75-1.0$.

\section{Results}

The 46 patients comprised 24 women and 22 men with a mean age of $60.2 \pm 10.8$ years, ranging from 28 76 years. Most patients received imaging for staging and restaging purposes. These patients had a history of one of the following types of neoplasms: breast-cancer $n=13$; lymphoma $\mathrm{n}=6$; gastrointestinal-cancer $\mathrm{n}=5$; lung-cancer $\mathrm{n}=4$; prostate-cancer $\mathrm{n}=3$; gynecological cancer of the pelvis $\mathrm{n}=3$; cancer-of-unknown-origin $\mathrm{n}=3$; head/neck-cancer $\mathrm{n}=2$; thyroid-cancer $\mathrm{n}=2$; renal-cancer $\mathrm{n}=1$ and liver-cancer $\mathrm{n}=1$. Two patients received imaging to investigate unclear weight loss and one patient due to unclear inflammation.

\section{Objective assessment}

As the corrected attenuation is the difference between artifact impaired and artifact-free reference tissue, $0 \mathrm{HU}$ 
Table 1 Visual analysis

Extent of hypo- and hyperdense artifacts

[5] Artifacts are absent or almost absent; [4] minor artifacts; [3] moderate artifacts; [2] pronounced artifacts; [1] massive artifacts

Diagnostic assessment of surrounding soft tissue (e.g., muscle, lymph nodes, and fat)

[5] Full diagnostic quality as there were no artifacts or almost no artifacts; [4] marginally affected diagnostic quality by minor streaks; [3] hampered diagnostic quality by moderate artifacts; [2] restricted diagnostic quality by strong artifacts; [1] insufficient diagnostic quality

Diagnostic assessment of vessels (e.g., axillary and subclavian artery and vein)

[5] Full diagnostic quality as no artifacts or almost no artifacts; [4] marginally affected diagnostic interpretability by minor streaks; [3] hampered diagnostic interpretability by moderate artifacts; [2] restricted diagnostic interpretability by strong artifacts; [1] insufficient diagnostic interpretability

Presence of overcorrection and new artifacts compared to $\mathrm{Cl}$

[3] No overcorrection or new artifacts; [2] overcorrection, new opposite artifacts in the location of initial artifacts without additional impairment of diagnostic assessment compared to $\mathrm{Cl}$; [1] new artifacts in the same or different location of initial artifacts with additional impairment of diagnostic assessment compared to $\mathrm{Cl}$

Monoenergetic level with best diagnostic assessment regarding artifact extent, introduction of overcorrection or new artifacts, loss of tissue and vessel contrast

$\mathrm{Cl}$, conventional images.

suggests elimination of the initial artifact. For hypodense artifacts, corrected attenuation higher than $0 \mathrm{HU}$ suggests overcorrection of the initial hypodense artifact; for hyperdense artifacts, corrected attenuation lower than $0 \mathrm{HU}$ suggests overcorrection. Corrected attenuation within most pronounced hypo- and hyperdense artifacts significantly increased and decreased, respectively, in ARA, $\mathrm{VMI} \geq 80 \mathrm{keV}$, and $\mathrm{VMI}_{\mathrm{ARA}}$ between 70-200 keV (Table 2, Figure 1). In hypodense artifacts, corrected attenuation was closest to zero at VMI between $110-120 \mathrm{keV}$ and turned positive for $\mathrm{VMI} \geq 120 \mathrm{keV}$ while corrected attenuation for $\mathrm{VMI}_{\mathrm{ARA}}$ was closest to zero at between $90-100 \mathrm{keV}$ and turned positive for $\mathrm{VMI}_{\mathrm{ARA}} \geq 100 \mathrm{keV}$. In hyperdense artifacts, corrected attenuation was closest to zero at VMI between $100-110 \mathrm{keV}$ and turned negative for $\mathrm{VMI} \geq 110 \mathrm{keV}$ while corrected attenuation for $\mathrm{VMI}_{\mathrm{ARA}}$ was closest to zero between $90-100 \mathrm{keV}$ and turned negative for $\mathrm{VMI}_{\mathrm{ARA}}$ $\geq 100 \mathrm{keV}$ (Table 2, Figure 1). For 70-110 keV, ARA combined with VMI offered additional benefit compared to VMI alone significantly improving hypo- and hyperdense artifacts $(\mathrm{P}<0.05)$. For $\geq 120 \mathrm{keV}$, the combination of ARA and VMI did not demonstrate additional artifact reduction beyond what was achieved with VMI alone $(\mathrm{P}>0.05)$.

Muscle and arteries surrounding perivenous artifacts were also impaired. Arteries were mostly affected by hyperdense artifacts on which we therefore focused our measurements. Muscle was affected by both hypo- and hyperdense artifacts, here we measured only in the hypodense artifacts so that both artifact types were measured in artifact impaired organs. In muscle affected by hypodense artifacts, corrected attenuation was closest to zero at VMI between $150-160 \mathrm{keV}$ and turned positive for $\mathrm{VMI} \geq 160 \mathrm{keV}$ while corrected attenuation for $\mathrm{VMI}_{\mathrm{ARA}}$ was closest to zero at between $140-150 \mathrm{keV}$ and turned positive for $\mathrm{VMI}_{\mathrm{ARA}} \geq 150 \mathrm{keV}$ (Table 2, Figure 2). For 70-130 keV, ARA combined with VMI offered additional benefit and significantly reduced artifacts in muscle compared to VMI alone $(\mathrm{P}<0.05)$. In arteries affected by hyperdense artifacts, corrected attenuation was closest to zero at VMI between $110-120 \mathrm{keV}$ and turned negative for $\mathrm{VMI} \geq 120 \mathrm{keV}$ while corrected attenuation for $\mathrm{VMI}_{\mathrm{ARA}}$ was closest to zero between $80-90 \mathrm{keV}$ and turned negative for $\mathrm{VMI}_{\mathrm{ARA}} \geq 90 \mathrm{keV}$ (Table 2, Figure 2). For 70-100 keV, ARA combined with VMI offered additional benefit and significantly reduced artifacts in arteries compared to VMI alone $(\mathrm{P}<0.05)$.

Corrected image noise significantly decreased in artifact impaired muscle in $\mathrm{ARA}, \mathrm{VMI} \geq 110 \mathrm{keV}$, and $\mathrm{VMI}_{\mathrm{ARA}}$ between $70-200 \mathrm{keV}$ and arteries in $\mathrm{VMI} \geq 160 \mathrm{keV}$ and $\mathrm{VMI}_{\text {ARA }} \geq 130 \mathrm{keV}$ (Table 2).

\section{Visual assessment}

Corresponding to the objective results, visual assessment 
Table 2 Objective assessment of artifact reduction and surrounding tissue

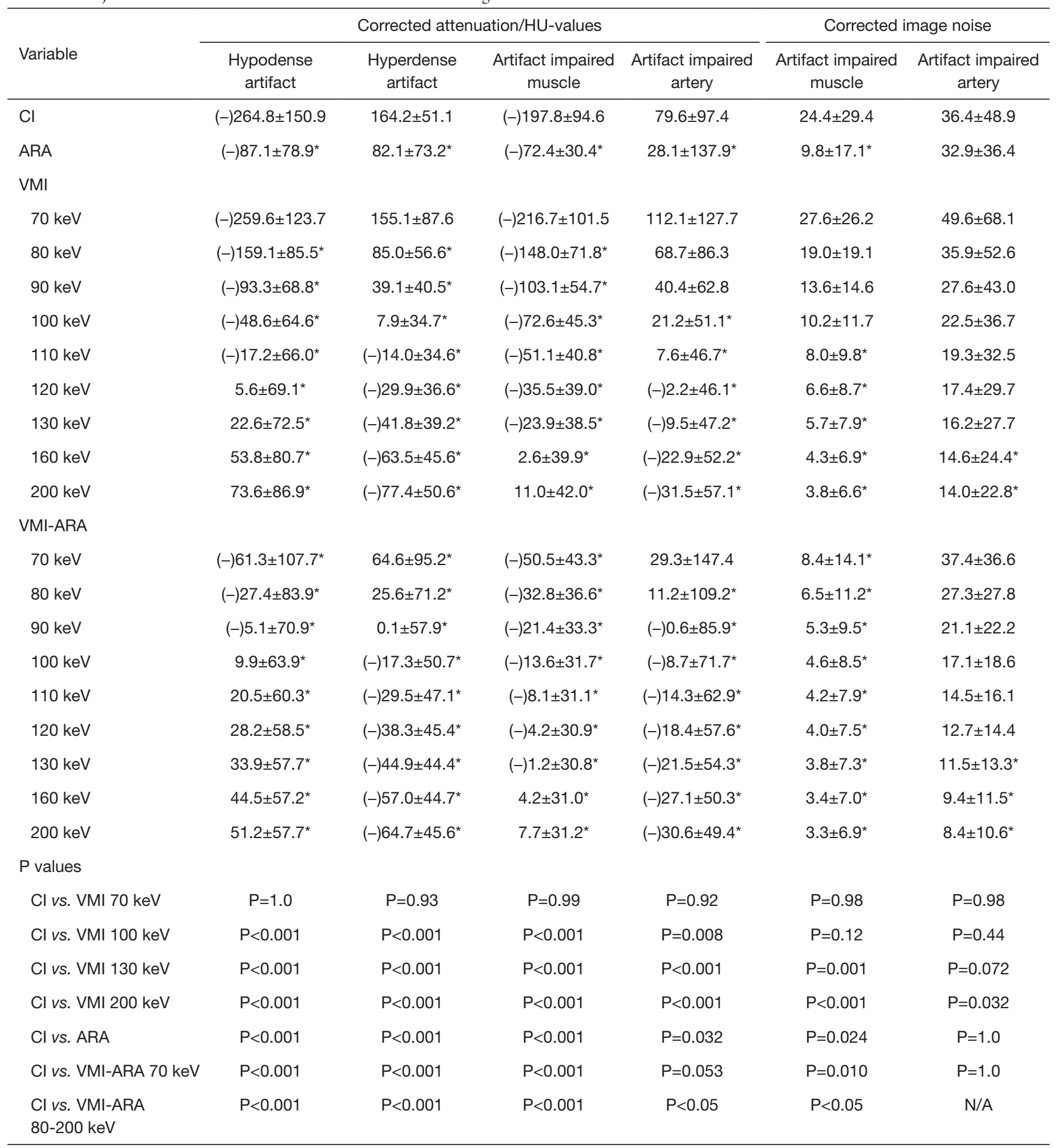

*, significant changes in $\mathrm{HU}$-values compared to $\mathrm{Cl}$ are marked $(\mathrm{P}<0.05)$. $\mathrm{Cl}$, conventional images; VMI, virtual monoenergetic images; ARA, artifact reduction algorithms; VMI-MAR, combination of ARA and VMI. 


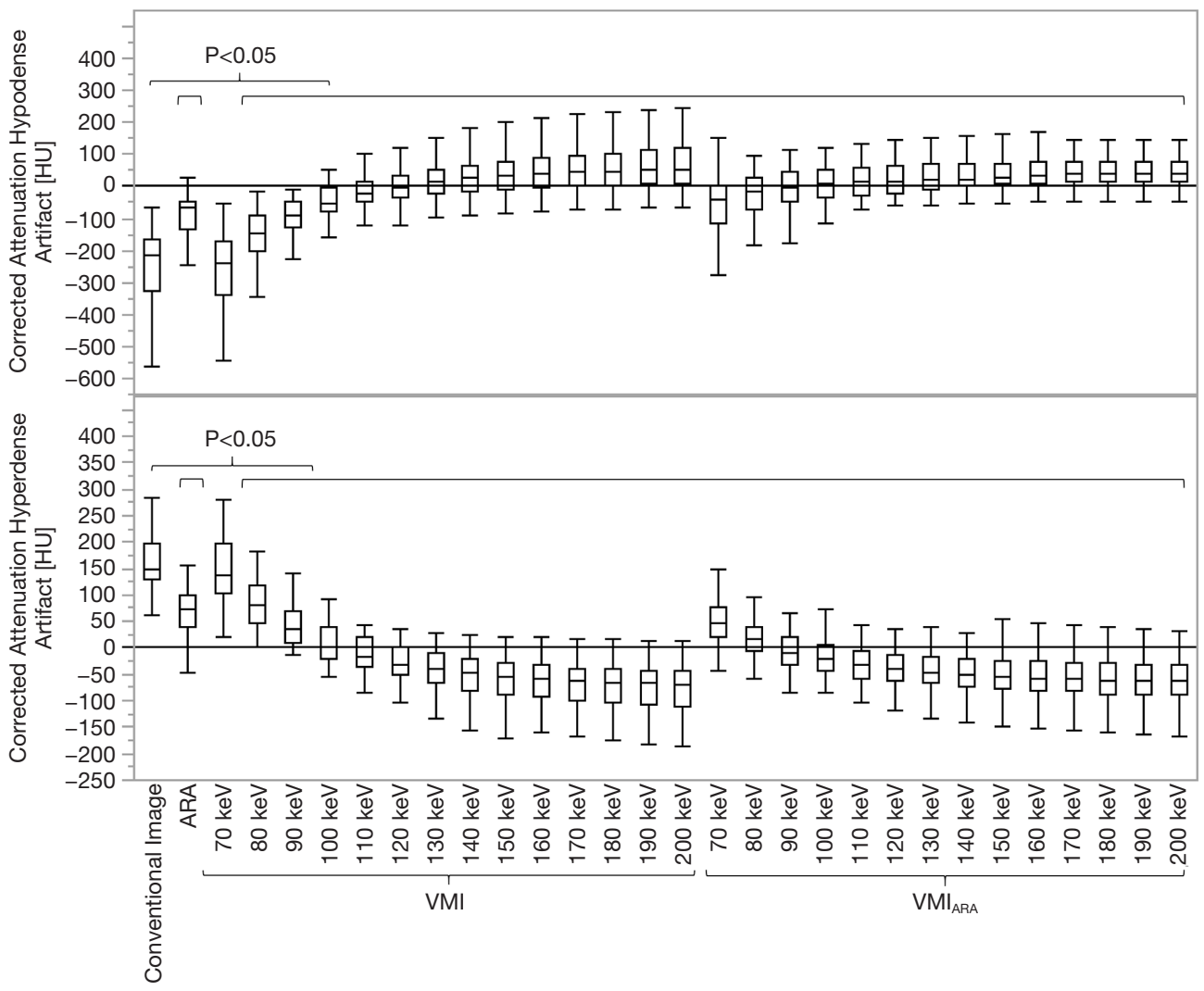

Figure 1 Box-plot diagram displaying corrected attenuation values within hypo- and hyperdense perivenous artifacts in conventional CT images, artifact reduction algorithms (ARA), virtual monoenergetic images (VMI, 70-200 keV), and their combination $\mathrm{VMI}_{\mathrm{ARA}}$. Hypo- and hyperdense artifacts showed significant improvement as evidenced by increasing and decreasing attenuation differences between artifact impaired and artifactfree reference tissue (corrected attenuation) in ARA, $\mathrm{VMI} \geq 80 \mathrm{keV}$, and $\mathrm{VMI}_{\mathrm{ARA}}$ between $70-200 \mathrm{keV}$, compared to conventional images.

of hypo- and hyperdense artifacts revealed significant reduction in ARA, VMI $\geq 100 \mathrm{keV}$, and $\mathrm{VMI}_{\mathrm{ARA}}$ between 70-200 keV (Table 3, Figures 3,4). Overcorrection of initial artifacts and new artifacts were reported in ARA, VMI, and $\mathrm{VMI}_{\mathrm{ARA}}$. The number of patients affected by overcorrection and new artifacts increased at higher $\mathrm{keV}$ values (Table 4, Figure 4A). Despite frequent overcorrection at higher $\mathrm{keV}$ values, diagnostic assessment by new artifacts compared to CI was mostly not additionally hampered by ARA, VMI and their combination. For VMI between $100-130 \mathrm{keV}$, additional impairment by new artifacts was not reported at all whereas for $\mathrm{VMI}_{\mathrm{ARA}}$ between $100-130 \mathrm{keV}$, it was reported in $\leq 10 \%$ of the cases (Table 4).

Diagnostic assessment of surrounding soft tissue (muscle, lymph nodes, and fat; Figure 3) significantly improved in ARA, VMI $\geq 100 \mathrm{keV}$ and $\mathrm{VMI}_{\mathrm{ARA}}$ between $70-200 \mathrm{keV}$ and was rated best in VMI at $130 \mathrm{keV}$ and $\mathrm{VMI}_{\mathrm{ARA}}$ at $100 \mathrm{keV}$
(Figure $4 B$ ). At higher $\mathrm{keV}$, introduction of overcorrection and new artifacts as well as loss of soft tissue contrast negatively affected soft tissue assessment (Figure $4 A$ ). Diagnostic assessment of surrounding vessels significantly improved in $\mathrm{VMI} \geq 100 \mathrm{keV}$ and $\mathrm{VMI}_{\mathrm{ARA}}$ between 70 $200 \mathrm{keV}$ and was rated best in VMI at $130 \mathrm{keV}$ and $\mathrm{VMI}_{\text {ARA }}$ at $130 \mathrm{keV}$. At higher $\mathrm{keV}$, loss of vessel contrast negatively affected assessment. ARA alone was not able to significantly improve diagnostic assessment of arteries.

Mean optimal $\mathrm{keV}$ values for diagnostic assessment were $127 \pm 18 \mathrm{keV}$ (range, $90-165 \mathrm{keV}$ ) for VMI and $116 \pm 21 \mathrm{keV}$ (range, 78-166 keV) for $\mathrm{VMI}_{\text {ARA }}$ (Figure 4). Optimal keV values were significantly lower for combination of ARA and VMI compared to VMI alone $(\mathrm{P}<0.05)$.

After applying ARA, VMI and/or $\mathrm{VMI}_{\mathrm{ARA}}$ ten additional lymph nodes could be revealed that were in CI obscured by artifacts (Figure 3). 


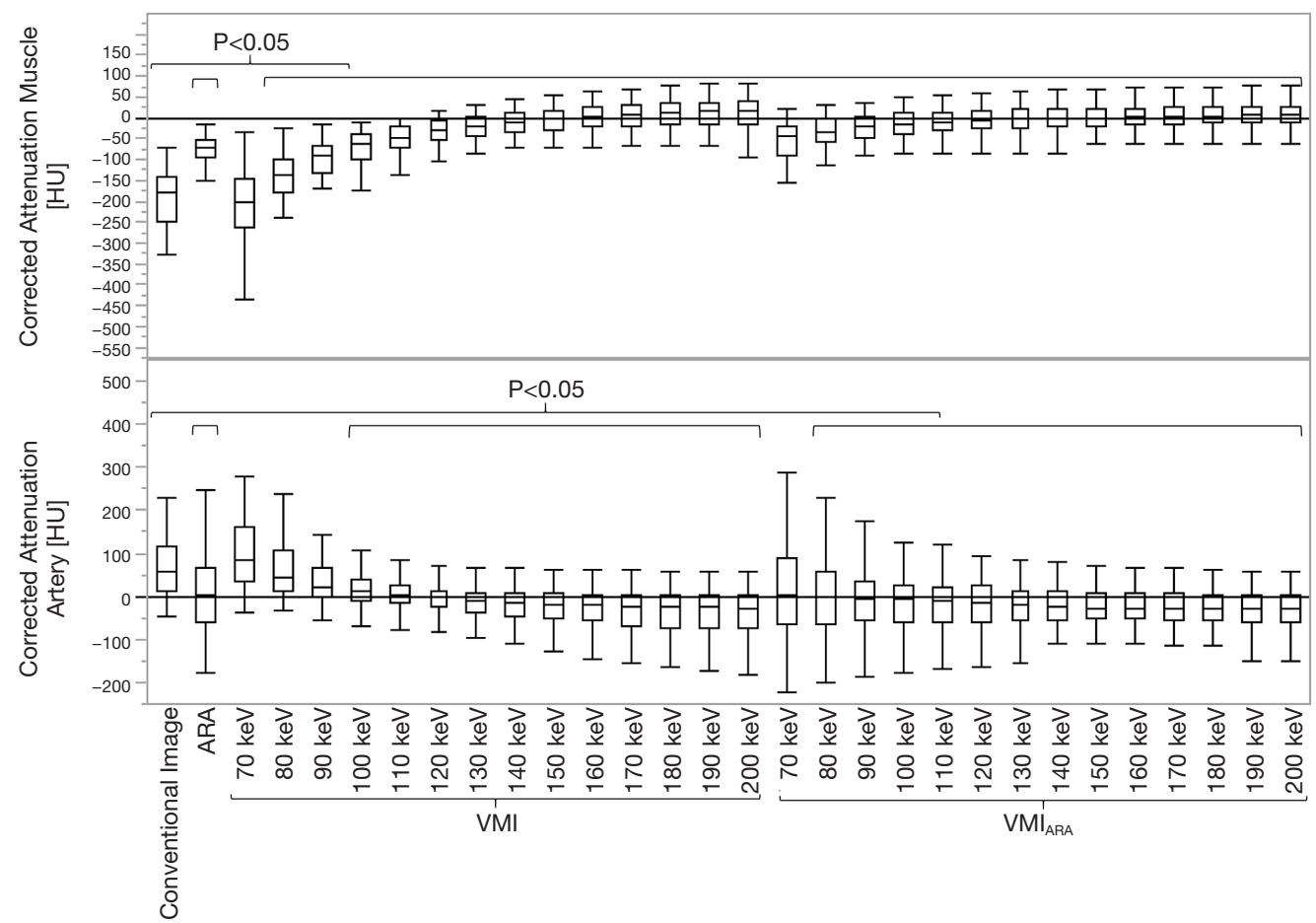

Figure 2 Box-plot diagram displaying corrected attenuation values within artifact impaired muscle and artery from perivenous artifacts in conventional CT images, artifact reduction algorithms (ARA), virtual monoenergetic images (VMI, 70-200 keV), and their combination $\mathrm{VMI}_{\mathrm{ARA}}$. By hypodense artifacts impaired muscle showed significant improvement as evidenced by increasing attenuation differences between artifact impaired and artifact-free reference tissue (corrected attenuation) in ARA, VMI $\geq 80 \mathrm{keV}$, and $\mathrm{VMI}_{\mathrm{ARA}}$ between $70-200 \mathrm{keV}$, compared to conventional images. By hyperdense artifacts impaired arteries showed significant improvement as evidenced by decreasing attenuation differences between artifact impaired and reference tissue in ARA, VMI $\geq 100 \mathrm{keV}$, and $\mathrm{VMI}_{\mathrm{ARA}}$ between $80-200 \mathrm{keV}$, compared to conventional images.

Overall interrater-agreement was excellent (ICC: 0.86, Table 3).

\section{Discussion}

This study assessed the value of ARAs and spectral detector CT-enabled virtual monoenergetic imaging for contrast media artifacts in chest imaging. After upper extremity injection these artifacts were most pronounced around axillary and subclavian veins. Objective results demonstrated all three techniques yielded a clear reduction of both, hypoand hyperdense artifacts. This was supported by subjective analysis that showed reduction of artifacts by ARA, VMI and their combination and thereby revealed an improved diagnostic assessment of adjacent soft tissue (e.g., muscle, lymph nodes, and fat). Assessment of surrounding vessels only improved in VMI and in the combination with ARA but not for ARA alone. Evaluation of chest staging and restaging examinations could benefit as visualization and detection of soft tissue and lymph node metastases as well as vessel pathologies might be facilitated (10,29,30).

Postprocessing based on ARA is available from all major vendors and has successfully been applied for artifact reduction in metal implants $(16,19,31)$. To our knowledge, this is the first study that reported the dedicated value of ARA alone and in combination with VMI for reduction of artifacts from contrast media. In concordance with current literature $(2,21,22)$, the combination of ARA and VMI and VMI as a standalone method were found most effective for reduction of artifacts as opposed to ARA alone. Still, there were some differences in performance between $\mathrm{VMI}_{\mathrm{ARA}}$ and VMI. The combination was significantly more effective than VMI alone between $70-110 \mathrm{keV}$. This offers the unique opportunity to receive optimal artifact 
Table 3 Subjective assessment of artifact reduction and diagnostic assessment

\begin{tabular}{|c|c|c|c|c|c|}
\hline Variable & \multicolumn{2}{|c|}{ Artifact extent } & \multicolumn{2}{|c|}{ Diagnostic assessment } & New artifacts \\
\hline $\mathrm{Cl}$ & $3[1-4]$ & 3 [2-4] & $3[1-4]$ & $2[1-4]$ & N/A \\
\hline ARA & $3[2-4]^{\star}$ & $3[2-4]^{*}$ & $3[2-5]^{\star}$ & $3[1-4]$ & 3 [2-3] \\
\hline \multicolumn{6}{|l|}{ VMI } \\
\hline $100 \mathrm{keV}$ & $4[3-5]^{*}$ & $4[3-5]^{*}$ & $4[3-5]^{\star}$ & $4[3-5]^{\star}$ & 3 [3-3] \\
\hline $130 \mathrm{keV}$ & $5[4-5]^{*}$ & $5[4-5]^{*}$ & $5[3-5]^{\star}$ & $5[4-5]^{*}$ & 3 [2-3] \\
\hline $160 \mathrm{keV}$ & $5[4-5]^{\star}$ & $5[4-5]^{*}$ & $4[2-5]^{\star}$ & $4[4-5]^{\star}$ & $2[1-3]$ \\
\hline $200 \mathrm{keV}$ & $5[5-5]^{*}$ & $5[4-5]^{*}$ & $4[2-5]^{\star}$ & $4[3-5]^{\star}$ & $2[1-3]$ \\
\hline $100 \mathrm{keV}$ & $5[4-5]^{\star}$ & $4[4-5]^{\star}$ & $5[3-5]^{*}$ & $4[3-5]^{*}$ & 3 [2-3] \\
\hline $130 \mathrm{keV}$ & $5[4-5]^{\star}$ & $5[3-5]^{*}$ & $4.5[2-5]^{\star}$ & $4.5[3-5]^{\star}$ & $2[1-3]$ \\
\hline $160 \mathrm{keV}$ & $5[5-5]^{\star}$ & $5[4-5]^{*}$ & $4[2-5]^{\star}$ & $4[3-5]^{\star}$ & $2[1-3]$ \\
\hline $200 \mathrm{keV}$ & $5[5-5]^{\star}$ & $5[4-5]^{\star}$ & $4[2-5]^{\star}$ & $4[3-5]^{\star}$ & $2[1-3]$ \\
\hline ICC & 0.90 & 0.89 & 0.80 & 0.77 & 0.55 \\
\hline \multicolumn{6}{|l|}{$P$ values } \\
\hline Cl vs. VMI $70 \mathrm{keV}$ & $P=0.98$ & $P=1.0$ & $P=1.0$ & $P=0.99$ & N/A \\
\hline Cl vs. VMI $100 \mathrm{keV}$ & $\mathrm{P}<0.001$ & $\mathrm{P}<0.001$ & $P<0.001$ & $\mathrm{P}<0.001$ & $\mathrm{~N} / \mathrm{A}$ \\
\hline Cl vs. VMI-ARA 100-200 keV & $\mathrm{P}<0.001$ & $\mathrm{P}<0.001$ & $P<0.001$ & $\mathrm{P}<0.001$ & $N / A$ \\
\hline
\end{tabular}

Data is reported as median and 10/90-percentile. * Significant changes in scores compared to $\mathrm{Cl}$ are marked $(\mathrm{P}<0.05)$. Vessels, axillary and subclavian vessels; soft tissue, adjacent soft tissue (e.g., muscle, lymph nodes, and fat); new artifacts, introduction of overcorrection or new artifacts compared to conventional images; CI, conventional images; VMI, virtual monoenergetic images; ARA, artifact reduction algorithms; VMI-ARA, combination of ARA and VMI; ICC, intraclass correlation.

reduction at relatively low $\mathrm{keV}$ values, where loss of tissue and vessel contrast is still low $(2,11,31,32)$ and introduction of overcorrection or new artifacts less common as revealed in our analysis and previous studies $(20,21)$. Mean optimal $\mathrm{keV}$ values for diagnostic assessment was $116 \mathrm{keV}$ for the combination of VMI and ARA, considerably lower than optimal $\mathrm{keV}$ values for VMI alone, $127 \mathrm{keV}$. At $\mathrm{keV}$ values of $\geq 120 \mathrm{keV} \mathrm{VMI}$ ARA and VMI showed comparable artifact reduction capabilities, e.g., $\mathrm{HU}$ values of $\mathrm{VMI}_{\mathrm{ARA}}$ at $120 \mathrm{keV}$ were comparable to VMI at $130 \mathrm{keV}$. Taking this into account that, $\mathrm{VMI}_{\mathrm{ARA}}$ at $120 \mathrm{keV}$ maintains higher soft tissue contrast but might show more overcorrection and artifacts, and VMI at $130 \mathrm{keV}$ should have a lower inherent soft tissue contrast but less overcorrection or new artifacts, each of the two approaches might be preferable depending on the individual imaging characteristics of the individual 


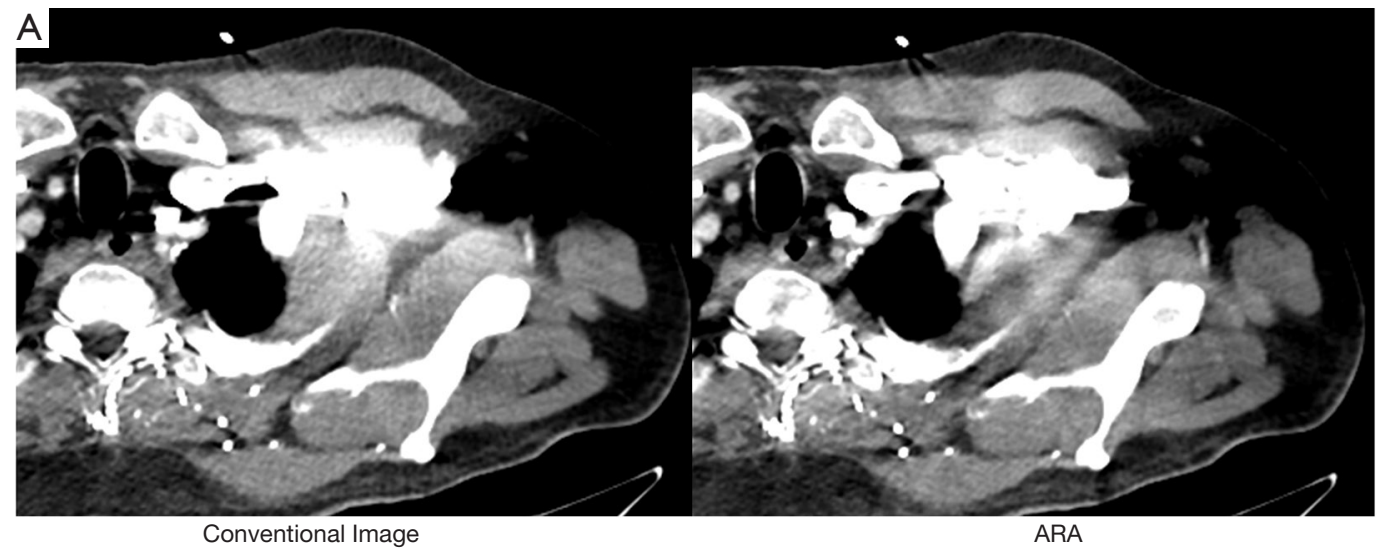

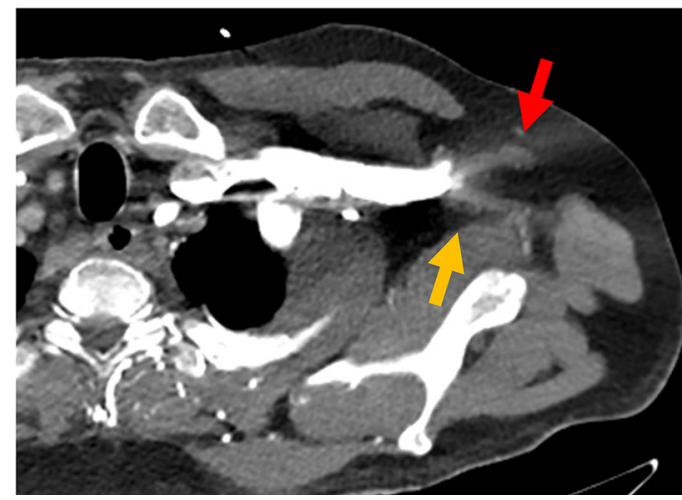

VMI $130 \mathrm{keV}$

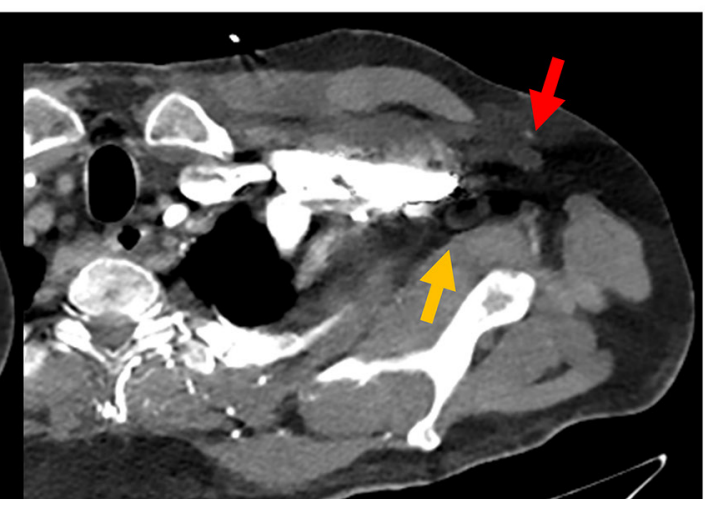

$\mathrm{VMI}_{\mathrm{ARA}} 130 \mathrm{keV}$

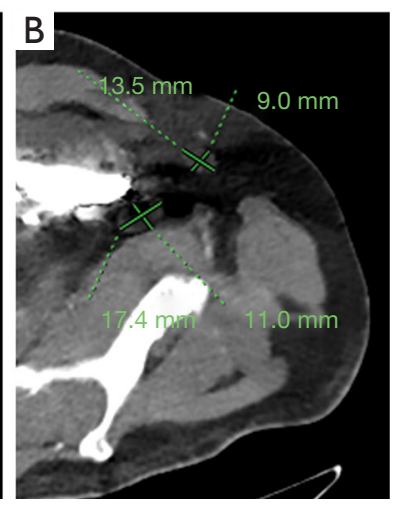

$\mathrm{VMI}_{\text {ARA }} 130 \mathrm{keV}$

Figure 3 Combination of ARA and VMI reveals two lymph nodes that were obscured by severe artifacts in CI. (A) CI, VMI at 130 keV, ARA reconstruction, and their combination $\left(\mathrm{VMI}_{\mathrm{ARA}}\right)$ at $130 \mathrm{keV}$ in soft tissue window settings (window level: 60, window width: 350 ) are presented of a 53-year-old male patient with known history of lymphoma. Only the combination of ARA and VMI at 130 keV depicts two left axillary lymph nodes (red and yellow arrows) that are obscured by a strong artifact in the conventional image. Of note, VMI at $130 \mathrm{keV}$ only shows the anterior lymph node (red arrow), while the other lymph node is not optimally seen (yellow arrow). (B) Zoomed in $\mathrm{VMI}_{\mathrm{ARA}}$ at $130 \mathrm{keV}$. ARA, artifact reduction algorithm; VMI, virtual monoenergetic image; CI, conventional CT image.

patients.

ARA as a single approach also reduced artifacts but was less effective compared to VMI and did not significantly improve visual assessment of surrounding vessels. Still, ARA alone has the advantage of increased availability compared to dual-energy CT $(16,19,20)$. Also, ARA do not suffer from a loss of contrast, which could be observed in our study in VMI at high keV and which has been reported in earlier studies $(2,31,32)$. Although the ARA used in this study is described as an algorithm for metal artifact reduction (20) our results additionally demonstrate reduced artifacts from highly concentrated boluses of contrast media which, like metal, are very highly attenuating in CT (20). It might be helpful in future studies to investigate possible adaption of ARA to properties of contrast media artifacts and evaluate if artifact reduction capabilities can be further enhanced.

The dedicated potential for VMI for reduction of artifacts from highly attenuating metals at various locations in the body and for multiple clinical indications (16,32-36) has been investigated for different clinically available dualenergy CT scanners. So far, reduction of artifacts from contrast media using VMI as a standalone method has been investigated by two recent studies establishing VMI as an effective method for reduction of these kinds of artifacts $(2,18)$. VMI are ideally suited to address contrast media artifacts since higher $\mathrm{keV}$ values yield an increased distance to the k-edge of iodine $(\sim 33 \mathrm{keV})$ and reduced attenuation from contrast (37-39). This was confirmed by our results which revealed effective reduction of hypo- and hyperdense artifacts by VMI. An advantage of VMI as a single approach 


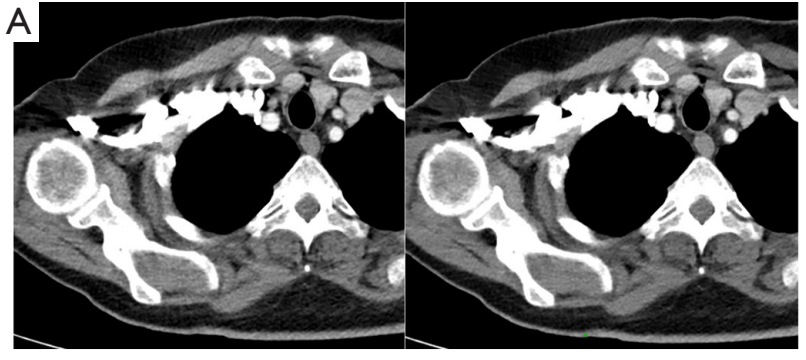

Conventional Image

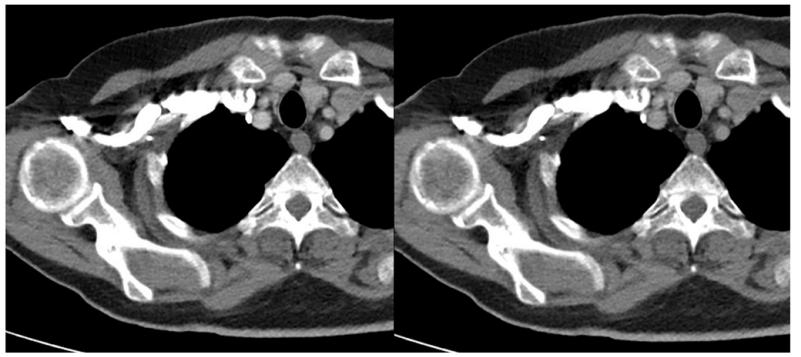

VMI 100 keV

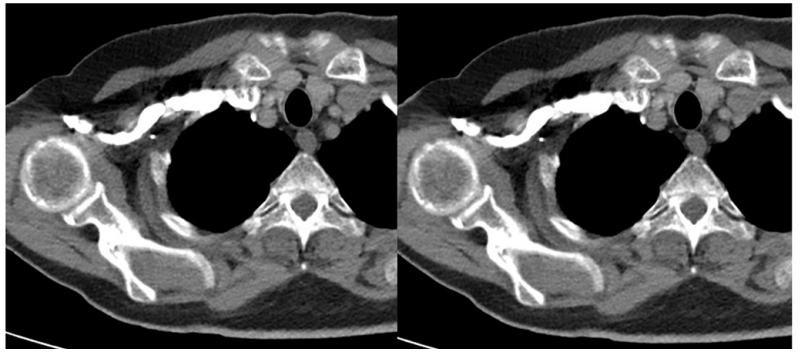

VMI 160 keV

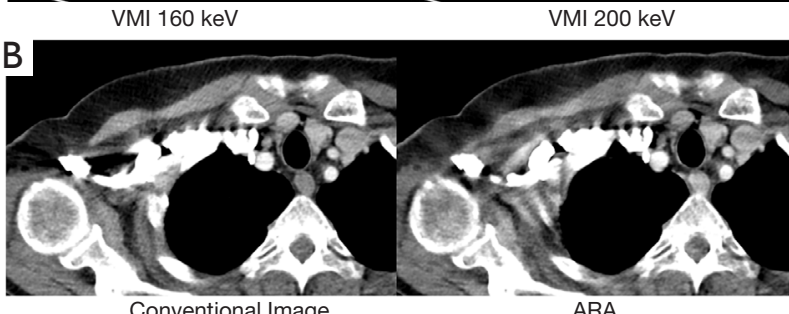

Conventional Image

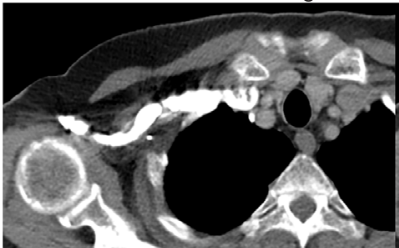

VMI $130 \mathrm{keV}$

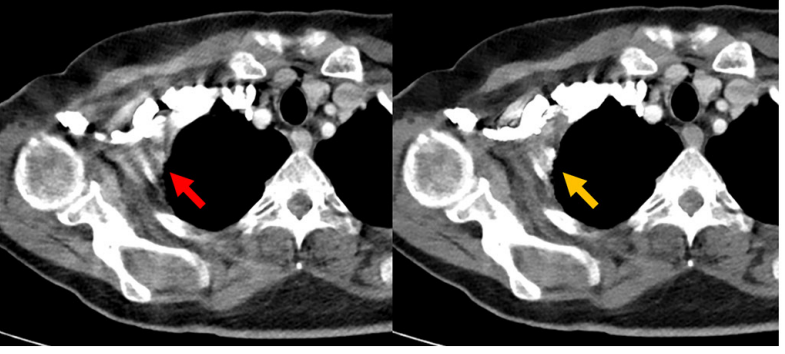

ARA

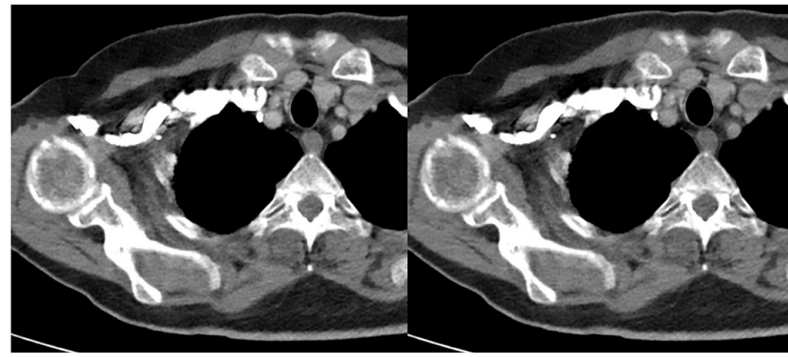

$\mathrm{VMI}_{\mathrm{ARA}} 100 \mathrm{keV}$

$\mathrm{VMI}_{\mathrm{ARA}} 130 \mathrm{keV}$

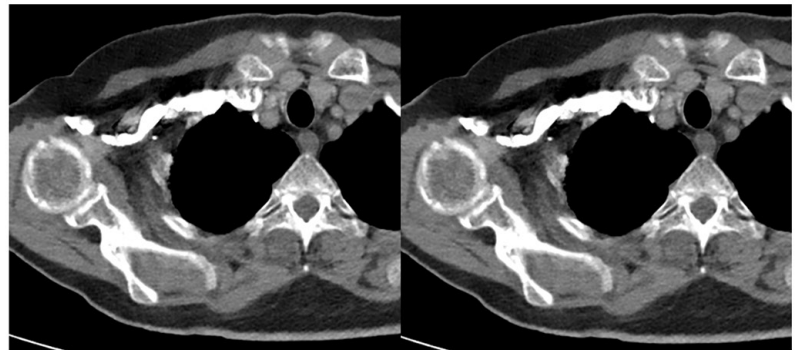

$\mathrm{VMI}_{\text {ARA }} 160 \mathrm{keV}$

Figure 4 Combination of ARA and VMI allow for a complete reduction of moderate artifacts after contrast media injection. (A) CI and virtual monoenergetic images (VMI, 70-200 keV), ARA reconstruction, and their combination $\left(\mathrm{VMI}_{\mathrm{ARA}}, 70-200 \mathrm{keV}\right)$ in soft tissue window settings (window level: 60, window width: 350) are presented of a 57-year-old female patient with renal cancer. ARA, VMI and their combination each allow for a strong reduction of artifacts, whereby artifact reduction is most effective in $\mathrm{VMI}_{\mathrm{ARA}}$ at $100 \mathrm{keV}$ and $\mathrm{VMI}$ at $130 \mathrm{keV}$. ARA introduces a new artifact by partially obscuring the rib posterior of the artifacts (red arrows). $\mathrm{VMI}_{\mathrm{ARA}}$ at $70 \mathrm{keV}$ has a better performance, as evidenced by a less strong impairment of the adjacent rib (yellow arrow). (B) Zoomed in images of CI, ARA, VMI at 130 $\mathrm{keV}$ and $\mathrm{VMI}_{\mathrm{ARA}}$ at $100 \mathrm{keV}$ of the same patient. ARA, artifact reduction algorithm; VMI, virtual monoenergetic image; CI, conventional CT image. 
Table 4 Overcorrection and new artifacts

\begin{tabular}{lccc}
\hline Variable & None & Overcorrection & New artifacts \\
\hline ARA & $78 \%(72 / 92)$ & $14 \%(13 / 92)$ & $8 \%(7 / 92)$ \\
VMI & & & $8 \%(2 / 92)$ \\
$70 \mathrm{keV}$ & $92 \%(85 / 92)$ & $5 \%(5 / 92)$ & $0 \%(0 / 92)$ \\
$100 \mathrm{keV}$ & $97 \%(89 / 92)$ & $3 \%(3 / 92)$ & $0 \%(0 / 92)$ \\
$130 \mathrm{keV}$ & $61 \%(56 / 92)$ & $39 \%(36 / 92)$ & $8 \%(14 / 92)$ \\
$160 \mathrm{keV}$ & $33 \%(30 / 92)$ & $52 \%(48 / 92)$ & $21 \%(19 / 92)$ \\
$200 \mathrm{keV}$ & $29 \%(27 / 92)$ & $50 \%(46 / 92)$ & $1 \%(1 / 92)$ \\
VMl-ARA & & & $3 \%(3 / 92)$ \\
$70 \mathrm{keV}$ & $79 \%(73 / 92)$ & $20 \%(18 / 92)$ & $10 \%(9 / 92)$ \\
$100 \mathrm{keV}$ & $63 \%(58 / 92)$ & $34 \%(31 / 92)$ & $18 \%(17 / 92)$ \\
$130 \mathrm{keV}$ & $48 \%(44 / 92)$ & $42 \%(39 / 92)$ & $26 \%(24 / 92)$ \\
$160 \mathrm{keV}$ & $36 \%(33 / 92)$ & $46 \%(42 / 92)$ & $41 \%(38 / 92)$ \\
$200 \mathrm{keV}$ & $33 \%(30 / 92)$ & & \\
\hline
\end{tabular}

Affected patients are reported in percentage and total count. $\mathrm{Cl}$, conventional images; VMI, virtual monoenergetic images; ARA, artifact reduction algorithms; VMI-ARA, combination of ARA and VMI; none, no overcorrection or new artifacts; overcorrection, new opposite artifact in the location of the initial artifact without additional impairment of diagnostic assessment compared to Cl; new artifacts, new artifacts in the same or different location of initial artifact with additional impairment of diagnostic assessment compared to $\mathrm{Cl}$.

is that it did not result in any new artifacts at 100 and $130 \mathrm{keV}$. Further, overcorrection compared to $\mathrm{VMI}_{\mathrm{ARA}}$ did also occur less often.

Optimization of injection protocols, such as applying a saline flush after contrast media injection, should be first choice to reduce artifacts from contrast media $(3,7,40)$. Despite best efforts contrast media artifacts can occur $(2,4,7)$. For example, cancer patients might be affected more often by an insufficient or not applied saline flush as they are frequently suffer from poor venous conditions $(7,10)$.

This study has the following limitations. First, objectively characterizing artifacts on CT-images, particularly VMI, is not straightforward. Most studies favor ROI-based measurement of mean and standard deviation of attenuation to characterize artifacts $(25,41,42)$. However, more complex methods can also be applied using artifact quantification by dedicated algorithms $(22,43)$. When considering artifact measurement from VMI it must be recognized that attenuation is different at each $\mathrm{keV}$ setting according to physical principles (11). Therefore, we applied an intraindividual comparison between artifact impaired tissue and corresponding artifact-free tissue, i.e., corrected image noise and corrected attenuation, as recently suggested and applied in subsequent studies $(25,26,44)$. It should also be considered that in the subjective analysis optimal $\mathrm{keV}$ values for diagnostic assessment $(116-127 \mathrm{keV})$ were at a level at which overcorrection already occurred, pointing out a potential limitation of the objective assessment. The ROI based assessment did not cover all parts of the artifacts equally as they were placed where artifacts were strongest. We noticed that in the area where the artifacts were initially strongest, overcorrection was more pronounced than in less strong parts of the artifacts. Further, for the measurement on the artifact impaired muscle only the hypodense artifacts were assessed and no muscle focused assessment of hyperdense artifacts was conducted. To address any limitations of the objective analysis and substantiate our results, we conducted a complementary subjective evaluation by two independent readers visually assessing artifact reduction and diagnostic assessment. Second, although contrast media concentration and injected volume were standardized, the severity of artifacts varied potentially due to individual differences in patient circulation, vessel anatomy and patient weight (causing differing injection duration). Third, our investigation was designed to serve as a proof of concept to evaluate performance of two so far only for metal artifact reduction established methods for highly attenuating contrast media. Therefore, we did 
not focus our investigation on a certain pathology and our results should be used as a starting point for more clinically focused studies in future.

\section{Conclusions}

In the presence of perivenous artifacts after injection into a brachial vein, we recommend combined use of ARA and VMI or VMI as a standalone method. Both methods demonstrated to work best at $\mathrm{keV}$ values between 100-130 $\mathrm{keV}$ where tissue contrast is still high and the probability of introducing new artifacts is lowest. Artifact reduction by ARA alone was significant but less effective. ARA and VMI as standalone approaches offer distinct advantages: ARA do not suffer from a loss of tissue or vessel contrast and are available with conventional CT whereas VMI yields stronger artifact reduction but requires a scanner with dualenergy CT capabilities.

\section{Acknowledgments}

Funding: This research was supported in part by Philips Healthcare under a research agreement with University Hospitals Cleveland Medical Center and Case Western Reserve University.

\section{Footnote}

Conflicts of Interest: All authors have completed the ICMJE uniform disclosure form (available at http://dx.doi. org/10.21037/qims-20-435). SL and DZ received research support from Philips Healthcare. NGH received speakers' honoraria from Philips Healthcare. The other authors have no conflicts of interest to declare.

Ethical Statement: This retrospective study was approved by the local institutional review board.

Open Access Statement: This is an Open Access article distributed in accordance with the Creative Commons Attribution-NonCommercial-NoDerivs 4.0 International License (CC BY-NC-ND 4.0), which permits the noncommercial replication and distribution of the article with the strict proviso that no changes or edits are made and the original work is properly cited (including links to both the formal publication through the relevant DOI and the license). See: https://creativecommons.org/licenses/by-nc-nd/4.0/.

\section{References}

1. Fleischmann D. Use of high-concentration contrast media in multiple-detector-row CT: principles and rationale. Eur Radiol 2003;13:M14-20.

2. Kim C, Kim D, Lee KY, Kim H, Cha J, Choo JY, Cho PK. The Optimal Energy Level of Virtual Monochromatic Images From Spectral CT for Reducing Beam-Hardening Artifacts Due to Contrast Media in the Thorax. AJR Am J Roentgenol 2018;211:557-63.

3. Haage P, Schmitz-Rode T, Hübner D, Piroth W, Günther RW. Reduction of Contrast Material Dose and Artifacts by a Saline Flush Using a Double Power Injector in Helical CT of the Thorax. AJR Am J Roentgenol 2000;174:1049-53.

4. Secchi F, De Cecco CN, Spearman JV, Silverman JR, Ebersberger U, Sardanelli F, Schoepf UJ. Monoenergetic extrapolation of cardiac dual energy CT for artifact reduction. Acta radiol 2015;56:413-8.

5. Mori I, Machida Y, Osanai M, Iinuma K. Photon starvation artifacts of X-ray CT: their true cause and a solution. Radiol Phys Technol 2013;6:130-41.

6. Boas FE, Fleischmann D. CT artifacts: causes and reduction techniques. Imaging Med 2012;4:229-40.

7. Fleischmann D. How to design injection protocols for multiple detector-row CT angiography (MDCTA). Eur Radiol 2005;15:E60-5.

8. Fayad LM, Patra A, Fishman EK. Value of 3D CT in Defining Skeletal Complications of Orthopedic Hardware in the Postoperative Patient. AJR Am J Roentgenol 2009;193:1155-63.

9. Lee MJ, Kim S, Lee SA, Song HT, Huh YM, Kim DH, Han SH, Suh JS. Overcoming Artifacts from Metallic Orthopedic Implants at High-Field-Strength MR Imaging and Multi-detector CT. Radiographics 2007;27:791-803.

10. Takeyama N, Ohgiya Y, Hayashi T, Takahashi T, Takasu D, Nakashima J, Kato K, Kinebuchi Y, Hashimoto T, Gokan T. Comparison of different volumes of saline flush in the assessment of perivenous artefacts in the subclavian vein during cervical CT angiography. Br J Radiol 2011;84:427-34.

11. McCollough CH, Leng S, Yu L, Fletcher JG. Dual- and Multi-Energy CT: Principles, Technical Approaches, and Clinical Applications. Radiology 2015;276:637-53.

12. Johnson TRC. Dual-energy CT: general principles. AJR Am J Roentgenol 2012;199:S3-8.

13. Alvarez RE, Macovski A. Energy-selective reconstructions in X-ray computerized tomography. Phys Med Biol 
1976;21:733-44.

14. Flohr TG, McCollough CH, Bruder H, Petersilka M, Gruber K, Sü $\beta$ C, Grasruck M, Stierstorfer K, Krauss B, Raupach R, Primak AN, Küttner A, Achenbach S, Becker C, Kopp A, Ohnesorge BM. First performance evaluation of a dual-source CT (DSCT) system. Eur Radiol 2006;16:256-68.

15. Lewis M, Reid K, Toms AP. Reducing the effects of metal artefact using high $\mathrm{keV}$ monoenergetic reconstruction of dual energy CT (DECT) in hip replacements. Skeletal Radiol 2013;42:275-82.

16. Wellenberg RHH, Hakvoort ET, Slump CH, Boomsma MF, Maas M, Streekstra GJ. Metal artifact reduction techniques in musculoskeletal CT-imaging. Eur J Radiol 2018;107:60-9.

17. Große Hokamp N, Laukamp KR, Lennartz S, Zopfs D, Abdullayev N, Neuhaus VF, Maintz D, Borggrefe J. Artifact reduction from dental implants using virtual monoenergetic reconstructions from novel spectral detector CT. Eur J Radiol 2018;104:136-42.

18. Laukamp KR, Gupta A, Große Hokamp N, Obmann VC, Graner FP, Ho V, Ros P, Ramaiya N, Gilkeson R. Role of spectral-detector CT in reduction of artifacts from contrast media in axillary and subclavian veins: single institution study in 50 patients. Acta Radiol 2020;61:450-60.

19. Huang JY, Kerns JR, Nute JL, Liu X, Balter PA, Stingo FC, Followill DS, Mirkovic D, Howell RM, Kry SF. An evaluation of three commercially available metal artifact reduction methods for CT imaging. Phys Med Biol 2015;60:1047-67.

20. Philips CT Clinical Science PHU. Metal Artifact Reduction for Orthopedic Implants (O-MAR) [Philips NetForum Community]. Jan 8, 2012. Available online: http://clinical.netforum.healthcare.philips.com/us_en/ Explore/White-Papers/CT/Metal-Artifact-Reductionfor-O

21. Laukamp KR, Zopfs D, Lennartz S, Pennig L, Maintz D, Borggrefe J, Große Hokamp N. Metal artifacts in patients with large dental implants and bridges: combination of metal artifact reduction algorithms and virtual monoenergetic images provides an approach to handle even strongest artifacts. Eur Radiol 2019;29:4228-38.

22. Große Hokamp N, Hellerbach A, Gierich A, Jordan DW, Visser-Vandewalle V, Maintz D, Haneder S. Reduction of Artifacts Caused by Deep Brain Stimulating Electrodes in Cranial Computed Tomography Imaging by Means of Virtual Monoenergetic Images, Metal Artifact Reduction
Algorithms, and Their Combination. Invest Radiol 2018;53:424-31.

23. Neuhaus V, Grosse Hokamp N, Zopfs D, Laukamp K, Lennartz S, Abdullayev N, Maintz D, Borggrefe J. Reducing artifacts from total hip replacements in dual layer detector CT: Combination of virtual monoenergetic images and orthopedic metal artifact reduction. Eur J Radiol 2019;111:14-20.

24. Bamberg F, Dierks A, Nikolaou K, Reiser MF, Becker CR, Johnson TRC. Metal artifact reduction by dual energy computed tomography using monoenergetic extrapolation. Eur Radiol 2011;21:1424-9.

25. Laukamp KR, Große Hokamp N, Alabar O, Obmann VC, Lennartz S, Zopfs D, Gilkeson R, Ramaiya N, Gupta A. Metal artifacts from sternal wires: evaluation of virtual monoenergetic images from spectral-detector CT for artifact reduction. Clin Imaging 2020;60:249-56.

26. Laukamp KR, Zopfs D, Wagner A, Lennartz S, Pennig L, Borggrefe J, Ramaiya N, Große Hokamp N. CT artifacts from port systems: Virtual monoenergetic reconstructions from spectral-detector CT reduce artifacts and improve depiction of surrounding tissue. European Journal of Radiology 2019;121:108733.

27. Cohen J. A Coefficient of Agreement for Nominal Scales. Educ Psychol Meas 1960;20:37-46.

28. Fleiss JL, Cohen J. The Equivalence of Weighted Kappa and the Intraclass Correlation Coefficient as Measures of Reliability. Educ Psychol Meas 1973;33:613-9.

29. Ong N, George M, Dutta R, Ng CH. CT imaging features of skeletal muscle metastasis. Clin Radiol 2019;74:374-7.

30. James J, Teo M, Ramachandran V, Law M, Stoney D, Cheng M. A critical review of the chest CT scans performed to detect asymptomatic synchronous metastasis in new and recurrent breast cancers. World J Surg Oncol 2019;17:40.

31. Laukamp KR, Lennartz S, Neuhaus VF, Große Hokamp N, Rau R, Le Blanc M, Abudullayev N, Mpotaris A, Maintz D, Borggrefe J. CT metal artifacts in patients with total hip replacements: for artifact reduction monoenergetic reconstructions and post-processing algorithms are both efficient but not similar. Eur Radiol 2018;28:4524-33.

32. Wellenberg RHH, Boomsma MF, van Osch JAC, Vlassenbroek A, Milles J, Edens MA, Streekstra GJ, Slump $\mathrm{CH}$, Maas M. Quantifying metal artefact reduction using virtual monochromatic dual-layer detector spectral CT imaging in unilateral and bilateral total hip prostheses. Eur J Radiol 2017;88:61-70.

33. Winklhofer S, Hinzpeter R, Stocker D, Baltsavias G, 
Michels L, Burkhardt J-K, Regli L, Valavanis A, Alkadhi H. Combining monoenergetic extrapolations from dualenergy CT with iterative reconstructions: reduction of coil and clip artifacts from intracranial aneurysm therapy. Neuroradiology 2018;60:281-91.

34. Cha J, Kim H-J, Kim ST, Kim YK, Kim HY, Park GM. Dual-energy CT with virtual monochromatic images and metal artifact reduction software for reducing metallic dental artifacts. Acta Radiol 2017;58:1312-9.

35. Higashigaito K, Angst F, Runge VM, Alkadhi H, Donati OF. Metal Artifact Reduction in Pelvic Computed Tomography With Hip Prostheses: Comparison of Virtual Monoenergetic Extrapolations From Dual-Energy Computed Tomography and an Iterative Metal Artifact Reduction Algorithm in a Phantom Study. Invest Radiol 2015;50:828-34.

36. Dong Y, Shi AJ, Wu JL, Wang RX, Sun LF, Liu AL, Liu YJ. Metal artifact reduction using virtual monochromatic images for patients with pedicle screws implants on CT. Eur Spine J 2016;25:1754-63.

37. Bushberg JT. The AAPM/RSNA physics tutorial for residents. X-ray interactions. Radiographics 1998;18:457-68.

38. Riederer SJ, Mistretta CA. Selective iodine imaging using $\mathrm{K}$-edge energies in computerized x-ray tomography. Med Phys 1977;4:474-81.

39. Manjunatha HC, Rudraswamy B. Study of effective atomic number and electron density for tissues from human

Cite this article as: Gupta A, Obmann VC, Jordan M, Lennartz S, Obmann MM, Große Hokamp N, Zopfs D, Pennig L, Fürtjes G, Ramaiya N, Gilkeson R, Laukamp KR. CT artifacts after contrast media injection in chest imaging: evaluation of post-processing algorithms, virtual monoenergetic images and their combination for artifact reduction. Quant Imaging Med Surg 2021;11(1):226-239. doi: 10.21037/qims-20435 organs in the energy range of $1 \mathrm{keV}-100 \mathrm{GeV}$. Health Phys 2013;104:158-62.

40. Budoff MJ, Shinbane JS, Child J, Carson S, Chau A, Liu SH, Mao S. Multiphase Contrast Medium Injection For Optimization Of Computed Tomographic Coronary Angiography. Acad Radiol 2006;13:159-65.

41. Kidoh M, Nakaura T, Nakamura S, Tokuyasu S, Osakabe H, Harada K, Yamashita Y. Reduction of dental metallic artefacts in CT: Value of a newly developed algorithm for metal artefact reduction (O-MAR). Clin Radiol 2014;69:e11-6.

42. Bisschop R, Boomsma M, Van Raay JJA, Tiebosch ATM, Maas M, Gerritsma CL, Maas M, Bisschop R, Boomsma M, Raay J, Tiebosch A, Maas M, Gerritsma C, Boomsma M, Edens M, Lingen C Van, Warringa N, Ettema H, Verheyen C, et al. High Prevalence of Pseudotumors in Patients with a Birmingham Hip Resurfacing Prosthesis. J Bone Joint Surg Am 2013;95:1554-60.

43. Mangold S, Gatidis S, Luz O, König B, Schabel C, Bongers MN, Flohr TG, Claussen CD, Thomas C. Single-source dual-energy computed tomography: use of monoenergetic extrapolation for a reduction of metal artifacts. Invest Radiol 2014;49:788-93.

44. Laukamp KR, Ho V, Obmann VC, Herrmann K, Gupta A, Borggrefe J, Lennartz S, Große Hokamp N, Ramaiya N. Virtual non-contrast for evaluation of liver parenchyma and vessels: results from 25 patients using multi-phase spectral-detector CT. Acta Radiol 2020;61:1143-52. 